\title{
Société Française d'Allergie Séance du 20 Juin 1961
}

Précipińnes aux extraits de foin et de moisissures dans le serum des patients atteints de «farmer's lung». - M. J. Pepys.

L'étude des précipitines obtenues par la méthode de la diffusion sur gélose, en testant le serum de malades atteints de poumon de fermier avec des extraits de foin sain, de foin moisi et de diverses moisissures a permis de montrer qu'il existait dans le foin moisi un antigène provenant de $\Gamma$ action des moisissures sur le foin et que c'était cet antigène qui était responsable de la maladie. L'extrait de foin sain, les extraits des diverses moisissures considérés isolément ne contiennent pas cet antigène et sont incapables de provoquer la maladie. L'ensemencement de foin sain préalablement autoclave, avec diverses moisissures a permis d'obtenir expérimentalement un foin moisi qui avait les mêmes propriétés antigèniques que le foin moisi naturel. Ces etudes apportent pour la premiere fois la preuve sérologique d'une relation entre le foin moisi et la maladie dite Poumon de Fermier (Farmer's Lung).

Mise en evidence et dosage des anticorps allergiques par Thémagglutination «in vitro» dans les syndromes allergiques humains et expérimentaux.

MM. B. N. Halpern, M. Jacob, R. Binaghi et J. Parlebas décrivent une technique

d'hémagglutination passive permettant de doser in vitro les anticorps allergiques presents dans le serum.

L'étude a porté sur des serums d'animaux sensibilisés expérimentalement à diverses protéines et sur des serums d'individus spontanément allergiques soit à des pneumaller-gènes, soit à des protéines alimentaires.

La technique est d'une grande sensibilité: elle est positive avec les immunsérums expérimentaux jusqu'à un taux de dilution au millionième et avec les serums de malades allergiques jusqu'à une dilution au millième. La quantité minimum d'anticorps décelable par cette méthode est de 0,002 $\mu \mathrm{g} \mathrm{N} / \mathrm{ml}$.

La technique d'hémagglutination est spécifique car Гépuisement préalable des anticorps de

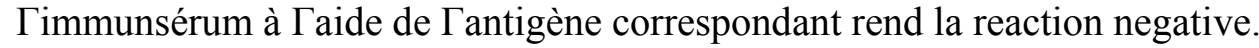

Les auteurs ont étudié les correlations entre le titre d'hémagglutinines et d'une part la dilution de Гantigène qui, en injection intradermique donne une reaction positive, d'autre part la dilution de Гimmunsérum qui donne, en transfert passif, une reaction positive. Ils discutent $\Gamma$ intérêt, les avantages et les applications de cette technique que sa complexité ne permet pas de considérer comme un procédé de diagnostic courant.

Le role des facteurs microbiens en tant qu'allergènes

MM. R. Wolpromm, N. T. Ky et E. Kaufman montrent combien il est difficile d'affirmer qu'un germe microbien agit en tant qu'allergène. Les tests cutanés sont aussi souvent positifs chez les témoins non allergiques que chez les allergiques. Pour afnrmer

252 Proceedings - Gesellschaftsberichte - Sociétés

Tallergie microbienne, il faut que Tallergie cutanée puisse être démontrée avec une très faible quantité de germes microbiens et surtout que Гinjection d'une dose minime de germes microbiens et surtout que Гinjection d'une dose minime d'allergène provoque une reaction 
focale nette. Le traitement est difficile car la dose minima efficace est toujours très proche de la dose maxima tolérée. Sur 105 malades allergiques infectés ce n'est que dans 6 cas (4 asthmes et 2 conjonctivités) que Tallergie microbienne a pu être démontrée et traitée avec succès.

Uallergie microbienne en ophtalmologie (d'après 65 observations). - N. T. Ky, Cl. Laroche, G. Offret, R. Campinchi, Mme Kastler.

Des recherches d'allergie microbienne ont été pratiquées chez 65 malades atteints d'aflections oculaires. Seul le streptocoque a donné des reactions cutanées importantes et prolongées. La reaction locale, même intense et retardée, n'a qu'une valeur d'orien-tation et ce sont les reactions générales et surtout focales, qui ont permis de retenir I'étiologie allergique dans 7 cas d'uvéite, 2 cas de kératite, 3 cas de cellulite orbitaire et 4 cas de conjonctivité.

Ces recherches paraissent particulièrement intéressantes dans les uvéites et les kératites dont I'étiologie reste inconnue dans la grande majorité des cas.

L'ædème de Qiiincke par allergie microbienne. (Analyse étiologique et thérapeutique de 61 observations).

MM. J. Blamoutier, L. Guibert et Cl. Laroche étudient 61 observations de malades présentant un cedème de Quincke chez lesquels la preuve a été faite par des arguments d'ordre clinique, biologique et thérapeutique que leur affection était due à une sensibilisation microbienne. Les antecedents infectieux étaient frequents surtout dans les jours précédant les poussées d'cedème. Mais parfois ils n'ont pu être mis en evidence que par un examen clinique attentif ou un examen de spécialistes: foyers otorhino-laryngologiques (49 cas), dermatologiques (20), gastroentérologiques (15), bronchiques (13), stomatologiques (13), urinaires (5), gynécologiques (3). Les germes dont le role allergisant a été prouvé par les reactions focales ont été le streptocoque (27 cas), le staphylocoque (16), Tanatoxine staphylococcique (10), le pneumocoque (5), le colibacille (3), le proteus (2). Chez 14 malades qui ne présentaient que des crises espacées, le traitement a porté sur le foyer infectieux seul. Les 47 autres ont subi un traitement de désensibilisation, associé dans 17 cas aux antibiotiques.

Les facteurs d'intolerance aux antibiotiques

MM. Cl. Huriez, P. Martin, et R. Fossati dans 100 cas d'intolérance médica-menteuse n'ont pu faire que 46 fois la preuve d'une sensibilisation. L'éosinophile n'était supérieure à $5 \%$ que dans 53 cas. Mais dans 75 cas le pouvoir histaminopexique du serum (PHP) était inférieur à 10\%, avec une valeur moyenne de $8 \%$, alors que les auteurs ont trouvé des valeurs moyennes de $8,9 \%$ pour 392 dermatoses allergiques certaines, $11,8 \%$ pour 85 dermatoses allergiques discutées et $25 \%$ pour 220 dermatoses réputées non allergiques et 80 témoins.

Dans ces intolerances, il fut observe une pénicillémie forte qu'il y avait intérêt à faire disparaître par injection de pénicillinase.

Chez des sujets sensibilisés, des concentrations particulièrement élevées lors de réinjections de pénicilline s'accompagnèrent de troubles électroencéphalographiques qui disparurent après Гélimination de la pénicilline.

En Tabsence de tests positifs, les eczemas et urticaires particulièrement rebelles après injection de certaines pénicillines-retard ou après emploi de penthotal ou d'évipan,

Proceedings - Gesellschaftsberichte - Sociétés 253

évoquent un blocage du système réticulo-endothélial dont les auteurs ont déjà démontré la réalité en mettant en evidence, par ponction biospie du foie, des granulations d'or et d'arsenic dans les cellules de Küpfer, des mois, voire des années après la dernière injection. 
Hormis les accidents de choc resultant d'un conflit antigène-anticorps, les auteurs insistent sur le fait que les intolerances médicamenteuses générales sont les mêmes chez les sujets sensibilisés que chez les non sensibilisés.

Collapsus après application co-saisonnière d'extrait de pollen sur un quadrillage cutane. - MM. J. Robin et P. Brevard.

Chez un sujet atteint de rhume des foins par allergie aux Graminées, déjà traité avec succès par cette méthode en 1960, le traitement est mis en æuvre cette année dès les premiers symptomes. Une demi-heure après la troisième application survient un collapsus brutal rapidement maîtrisé par une injection de corticoïde. Aucune raisori n'a pu être retenue, sinon que les pollens étaient ce jour là, particulièrement abondants. Cette méthode connaissant, en raison de sa simplicité, un grand succès, il était utile de rappeler que, pas plus qu'aucune autre méthode, elle n'offre une sécurité totale et ne doit être mise en æuvre que par des praticiens expérimentés. Vallergie de type retardé à la poussière de maison - 1 - Etude clinique et ventilatoire.

M. P. Burtin, Melle D. Buffe, M. J. Carton et Mme Dominjon-Monnier.

Les tests cutanés positifs d'horaire semi-retardé à la poussière de maison sont frequents, en particulier avec les fortes concentrations de cet extrait. Chez quelques mala-des, les reactions cutanées entraînent des reactions focales parfois sévères. Dans d'autres cas ce sont les tests ventilatoires qui ont mis en evidence une composante retardée plus ou moins importante dans Tallergie à la poussière de maison. Enfin des reactions in-solites au cours d'un traitement de désensibilisation peuvent aussi attirer Tattention.

II semble done exister une allergie de type retardé à la poussière de maison, pro-bablement liée à une substance différente de celle donnant $\Gamma$ allergie de type immédiat. Son mécanisme est discuté.

Uallergie de type retardé à la poussière de maison - 11 - Etude anatomo-pathologique des reactions cutanées.

Melle D. Buffe, MM. J. M. Verley, P. Burtin et P. Kourilsky.

Afin d'essayer de préciser le mécanisme immunologique de Tallergie de type re tardé à la Poussière de maison, des biopsies sur des reactions cutanées tardives ont été faites à des horaires variables (de 2 à 72 heures après $\Gamma$ intradermoréaction). 54 biopsies ont été ainsi pratiquées montrant des lesions d'un type constant: phase vasculo-exsudative d'abord, puis reaction cellulaire mononucléée. La signification de ces faits est discutée. J. Sglafer, Paris 